\title{
Gold Metal Liquid-Like Droplets
}

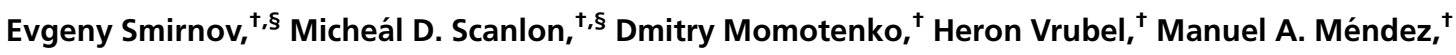 \\ Pierre-Francois Brevet, ${ }^{*}$ and Hubert H. Girault ${ }^{*,+}$
}

†Laboratoire d'Electrochimie Physique et Analytique, Ecole Polytechnique Fédérale de Lausanne, Station 6, CH-1015 Lausanne, Switzerland and ${ }^{\ddagger}$ Institut Lumière Matière, ILM UMR CNRS 5306, Universite Claude Bernard Lyon, 110 Rue Ada Byron, 69622 Villeurbanne Cedex, France. ${ }^{5}$ E. Smirnov and M. D. Scanlon contributed equally. The manuscript was written through contributions of all authors. All authors have given approval to the final version of the manuscript.

\begin{abstract}
Simple methods to self-assemble coatings and films encompassing nanoparticles are highly desirable in many practical scenarios, yet scarcely any examples of simple, robust approaches to coat macroscopic droplets with continuous, thick (multilayer), reflective and stable liquid nanoparticle films exist. Here, we introduce a facile and rapid one-step route to form films of reflective liquid-like gold that encase macroscopic droplets, and we denote these as gold metal liquid-like droplets (MeLLDs). The present approach takes advantage of the inherent self-assembly of gold nanoparticles at liquid-liquid interfaces and the increase in rates of nanoparticle aggregate trapping at the interface during emulsification. The ease of displacement of the stabilizing citrate ligands by appropriate redox active molecules that act as a lubricating molecular glue is key. Specifically, the heterogeneous interaction of citrate stabilized aqueous gold nanoparticles with the lipophilic electron donor tetrathiafulvalene under emulsified conditions produces gold MeLLDs. This methodology relies exclusively on electrochemical reactions, i.e., the oxidation of tetrathiafulvalene to its radical cation by the gold nanoparticle, and electrostatic interactions between the radical cation and nanoparticles. The gold MeLLDs are reversibly deformable upon compression and decompression and kinetically stable for extended periods of time in excess of a year.
\end{abstract}

KEYWORDS: gold nanoparticles · self-assembly · liquid-liquid interface · optical filter · liquid mirror

Fin. ilms and coatings of nanoparticles (NPs) are key ingredient components in many emerging technologies due to their distinctive optoelectrical, ${ }^{1,2}$ biological $^{3}$ and magnetic ${ }^{4}$ properties. Their remarkable utility has sparked huge interest in their potential applications as liquid mirrors, ${ }^{5}$ optical filters, ${ }^{6}$ sensors, ${ }^{7}$ catalysts, ${ }^{8}$ anticorrosion ${ }^{9}$ and antireflective $^{10}$ films, dialysis size selective membranes, ${ }^{11}$ photovoltaic light harvesters ${ }^{12}$ and antibacterial surfaces, ${ }^{13}$ among others.

The interface between two immiscible liquids, i.e., oil and water, is an extremely attractive scaffold at which to self-assemble NP films because of its defect-free pristine nature (facilitating reproducibly), transparency (advantageous for optical applications), self-healing dynamism (allowing self-assembly errors to be corrected rapidly) and mechanical flexibility (permitting planar, curved or 3D deformations). ${ }^{14,15}$ Self-assembly at liquidliquid interfaces is a classical bottom-up technique to produce $2 \mathrm{D}$ and $3 \mathrm{D}$ arrays and films of particles, especially metallic NPs. ${ }^{14,16,17}$

Since the pioneering work of Yogev and Efrima in $1988,{ }^{18}$ who described the formation of metal liquid-like films (MeLLFs), many methods have been introduced to self-assemble metallic NPs at air-liquid and liquid-liquid interfaces. Reported approaches include the addition of ethanol or methanol to the interfacial region, ${ }^{19,20}$ the use of salts or "promoters" 21,22 and covalent cross-linking interactions. ${ }^{11}$ An interesting approach by Han and co-workers involved displacing the stabilizing citrate ligands from the surface of colloidal gold nanoparticles (AuNPs) with either fullerene $\left(C_{60}\right)$ molecules $^{23}$ or carbon nanotubes (CNTs). ${ }^{24}$ With this approach they formed dense gold nanocomposite films at water-diethyl ether interfaces. Their proposed mechanism of film formation raised the possibility of charge transfer from the ligand (CNT or $\mathrm{C}_{60}$ ) to the AuNPs during the adsorption process. The end result of citrate displacement followed by charge transfer was the reduction in charge density on the surface of the AuNPs, a prerequisite for dense gold film formation. As discussed in more detail vide supra, it is possible that the CNTs and $\mathrm{C}_{60}$ molecules provide a "lubricating interfacial glue" layer that binds the AuNPs at the interface, and indeed, the majority *Address correspondence to
hubert.girault@epfl.ch.

Received for review July 4, 2014 and accepted September 3, 2014.

Published online September 03, 2014 $10.1021 / \mathrm{nn} 503644 \mathrm{v}$

() 2014 American Chemical Society 
of MeLLFs formed in the absence of a lubricating interfacial glue rapidly lose spectral reflectivity beyond monolayer surface coverage.

MeLLFs, however, are incapable of encapsulating macroscopic droplets. An inherent difficulty of encapsulating macroscopic droplets in continuous NP films is that NPs, as distinct from nanorods, ${ }^{25}$ lack "'surfactantlike"' symmetry to foster ordered, densely populated assemblies that are easily stabilized by noncovalent interactions alone on the interface. Kowalczyk et al. overcame this limitation by reinforcing noncovalent interactions with stabilizing cross-linkers between AuNPs functionalized with covalently attached selfassembled monolayers (SAMs) of 2,6-difluoro- $p$ mercaptophenol (DFMP). ${ }^{11}$ The partly hydrophobic character of the DFMP ligands (due to the fluorine groups) facilitated their ability to spread over a water-oil (toluene in this case) interface. When the cross-linker hexanedithiol was introduced to the organic phase the DFMP ligands at the droplet's surface were cross-linked creating a film of liquid-like gold around the aqueous droplet. The strong reflectivity and metallic gold luster of the film was indicative of bulk gold, and hence multilayer, formation. As these films encapsulate an oil droplet entirely, as opposed to simply forming a film between the oil and water phases, as is the case for traditional MeLLFs, ${ }^{26}$ we are denoting these films as rarely reported metal liquid-like droplets (MeLLDs). A radically different approach to forming gold MeLLDs was reported by Du et al., who coated millimeter sized liquid Gallium (Ga) droplets with layers of AuNPs stabilized by (1-mercaptoundec11-yl)-tetra(ethylene glycol) (TEG-OH) ligands. ${ }^{27,28}$ Irreversible adsorption of AuNPs minimized the large interfacial surface tension between $\mathrm{Ga}$ and the aqueous suspension by lowering the Ga-water contact area. Such gold MeLLDs remained smooth and reflective on the time scale of hours and the outer layer of AuNPs acted as a steric and/or electrostatic barrier to the coalescence of individual Ga droplets. ${ }^{27,28}$ To the authors knowledge, the only report to date that does not involve a covalent-bond induced stabilization procedure to form MeLLDs is that of Duan et al. ${ }^{29}$ who encapsulated toluene droplets in films of liquid-like gold using AuNPs capped with 2-bromo-2-methylpropionate ligands. Finally, some success in encapsulating microscopic droplets in NP films has been achieved utilizing diverse stabilizing strategies involving enzyme-AuNP polymeric type conjugates, ${ }^{30}$ coating the surface of the AuNPs with ligands consisting of mixed thiol monolayers, ${ }^{31}$ employing amphiphilic PEGylated AuNPs $^{32}$ or turning one phase into a gel. ${ }^{33}$ However, we do not designate such films as MeLLDs as these strategies are incapable of encasing macroscopic droplets.

Here, we introduce an approach to prepare gold MeLLDs that is facile, rapid and exclusive of covalent bond induced stabilization. Briefly, an aqueous colloidal

\section{(A)}

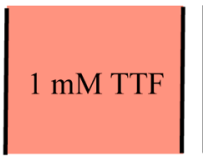

DCE

(B)

(i)

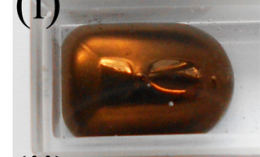

(ii)

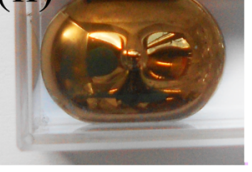

(E)
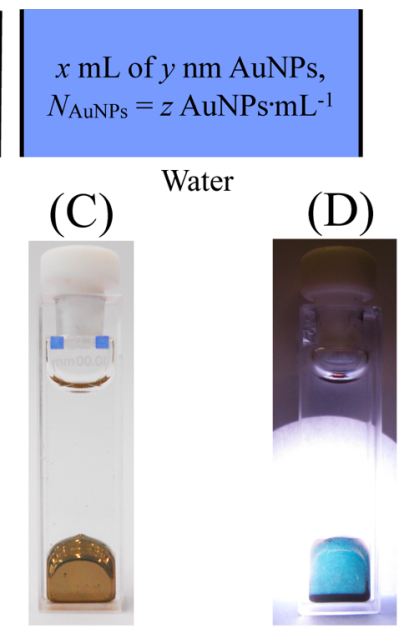

(D)

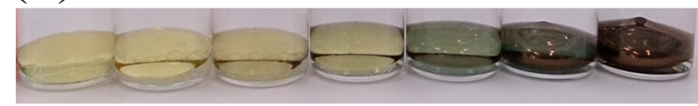

(F)

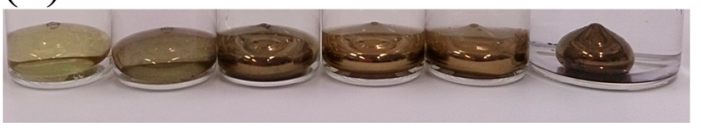

Figure 1. Reflective gold metal liquid-like droplets (MeLLDs). (A) Scheme of gold MeLLD formation, $1 \mathrm{~mL}$ of 1,2-dichoroethane (DCE) containing $1 \mathrm{mM}$ tetrathiafulvalene (TTF) was contacted with certain volumes $(x \mathrm{~mL})$ of colloidal AuNP solutions characterized by their average mean diameters, $d(y \mathrm{~nm})$, and number density of AuNPs, $N_{\text {AuNPs }}\left(z\right.$ AuNPs $\left.\cdot \mathrm{mL}^{-1}\right)$. Optical images of the gold MeLLDs formed by the (B) (i) smaller $\left(x=5, y=14, z=2.67 \times 10^{12}\right)$ and (B) (ii), (C) larger $\left(x=8, y=76, z=1.35 \times 10^{10}\right)$ AuNPs viewed in reflection mode; and (D) the smaller AuNPs $(x=3$, $y=14, z=2.67 \times 10^{12}$ ) viewed in transmission mode. The reflectance progressively increases with thicker films of the (E) smaller AuNPs ( $x=0,0.1,0.25,0.5,1,2$ and $5, y=14, z=$ $\left.2.67 \times 10^{12}\right)$ and $(F)$ larger AuNPs $(x=1,2,4,6,8$ and 35, $y=$ $\left.76, z=1.35 \times 10^{10}\right)$, from left to right.

AuNP solution is contacted with a 1,2-dichloroethane (DCE) oil phase incorporating tetrathiafulvalene (TTF, a sulfur containing lipophilic $\pi$-electron donor) and the system vigorously shaken with subsequent time given to settle (see Figure 1A). The completion time for the entire process ranges from $60 \mathrm{~s}$ for the smaller AuNPs up to $300 \mathrm{~s}$ for the larger AuNPs (see Movies S1 and S2 in the Supporting Information SI-1) and the film can be shaken, destroyed and reassembled ad infinitum (see Movies S3 and S4 in the Supporting Information SI-1).

\section{RESULTS AND DISCUSSION}

Optical Characterization of Gold MeLLDs. Two different gold MeLLDs were prepared with either relatively small (mean diameter of $14 \mathrm{~nm}$, as determined by characterization with microscopy, UV-vis spectroscopy and dynamic light-scattering (DLS) measurements, see Supporting Information SI-2 and SI-3) or large (mean diameter of $76 \mathrm{~nm}$ ) colloidal AuNP solutions. The gold MeLLDs were ellipsoidal in shape when formed but occasionally adopt a "teardrop" geometry due to either 

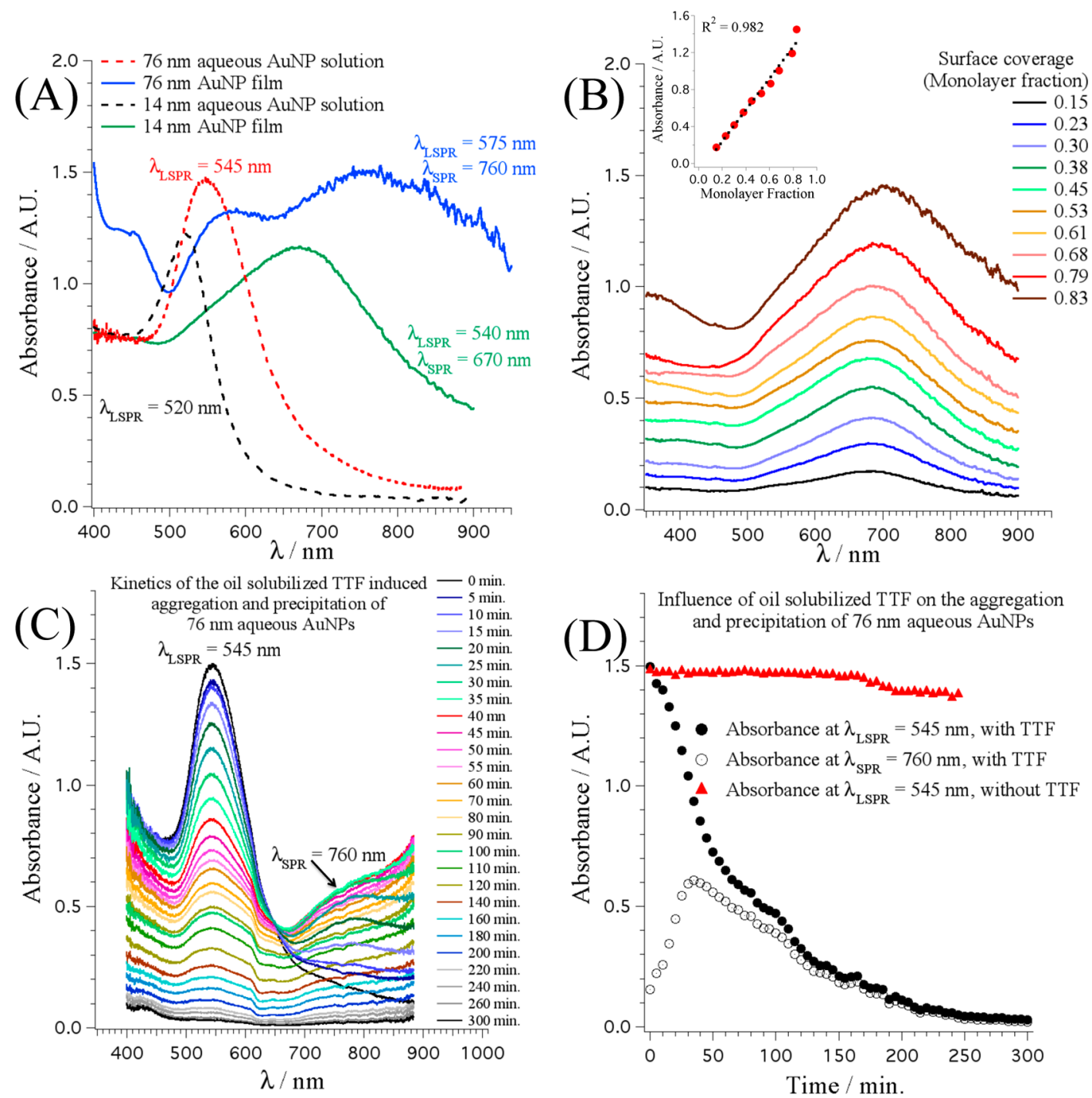

Figure 2. Characterization of the formation of gold MeLLDs. (A) Comparison of the extinction spectra of the aqueous AuNP colloidal solutions with the corresponding AuNP films of the MeLLDs ( 1 and $6 \mathrm{~mL}$ of colloidal solution were used for film formation with 14 and $76 \mathrm{~nm}$ AuNPs, respectively, see Figure 1A). (B) Monitoring the extinction spectra of the gold MeLLDs as a function of increasing AuNP surface coverage (expressed as a monolayer fraction assuming hexagonal close packing, see Supporting Information SI-4) for the $14 \mathrm{~nm}$ AuNPs. (C) Monitoring the kinetic behavior of the $76 \mathrm{~nm}$ AuNPs $\left(\lambda_{\mathrm{LSPR}}=545 \mathrm{~nm}\right)$ that aggregate $\left(\lambda_{\mathrm{SPR}} \approx 760 \mathrm{~nm}\right)$ and precipitate under stationary conditions on contact with TTF. Spectra of the water phase were recorded at regular 5 min intervals. (D) Comparison of the influence of the presence or absence of the electron donor TTF in the oil phase on the kinetics of AuNP aggregation and precipitation.

entrapment of an air bubble or the buildup of a bubble of evaporating DCE beneath the AuNP film. Such a teardrop geometry is clearly seen in Figure $1 \mathrm{E}, \mathrm{F}$, particularly at high AuNP surface coverages. Viewed in reflection mode, the multilayer films formed by 14 and $76 \mathrm{~nm}$ mean diameter AuNPs were reddish/brown (Figure 1B(i)) and gold (Figure 1B(ii); Figure 1C) in color, respectively. The latter is indicative of the presence of "bulk-like" behavior. Viewed in transmission mode the thinner reddish/brown films present nonvanishing blue transmissions, acting as blue filters (Figure 1D), by reducing the TTF concentration to micromolar levels in the oil droplet postfilm formation. These thinner films exhibit green colors with $1 \mathrm{mM}$ TTF in DCE, as expected from the complementary transmitted colors. The high reflectivity of MeLLDs is directly linked to the AuNP surface coverage (Figure 1E,F; see Movie S3 and Table S3 in the Supporting Information SI-4), whereas their metallic luster derives from their optical properties. $^{26,34-36}$

UV-vis characterization of both gold MeLLDs in transmission mode gave spectra with characteristic extinction peaks in the green/yellow and red regions (Figure 2A). Those in the green/yellow correspond to either the dipolar localized surface plasmon resonance (LSPR) of noninteracting AuNPs, or out-of-plane (transverse) AuNP plasmon modes for interacting AuNPs, at the water-oil interface. ${ }^{2,37}$ Meanwhile, those in the red region emerge from the in-plane (longitudinal) efficient coupling of the AuNP plasmon modes, arising 
from chains and islands of AuNPs present at submono-, mono- and multilayer film coverages. ${ }^{2,37}$ The latter arise from the collective excitation of free electrons of the AuNPs embedded in the film. ${ }^{2,37}$ Systematically increasing the quantity of AuNPs entrapped in a gold MeLLD (Figure 1E,F) revealed that the extinction peaks present at these higher wavelengths $(>650 \mathrm{~nm})$ are red-shifted and broadened (due to distance dependent plasmon coupling ${ }^{21}$ while their intensity increases in a linear fashion (Figure 2B). Schatz and co-workers ${ }^{38}$ developed an analytical model demonstrating that the plasmon wavelength shift is determined by the real part of the retarded dipole sum while the width is determined by the imaginary part of this sum. They discuss that optimal blue shifts and band narrowing are expected when the NP spacing is slightly smaller than the plasmon wavelength, while red shifts and broadening can be found for spacings much smaller than the plasmon wavelength at which electrostatic interactions are dominant. ${ }^{38}$ Herein, the latter expected red shifts and broadening were observed as the distance between AuNPs on the interface decreased to spacings much smaller than the plasmon wavelength with increasing surface coverage.

A series of control experiments were performed. First, the aqueous and DCE phases were studied by UV-vis spectroscopy pre- and postgold MeLLD formation and the spectra revealed no detectable traces of colloidal AuNPs in either phase postgold MeLLD formation (with both colloidal AuNP stock solutions). Thus, the vast majority (>99\%) of AuNPs were confined to the interfacial region and incorporated into the metallic film (see Figure S4 in the Supporting Information SI-5A). Second, the kinetics of TTF-induced AuNP aggregation were monitored by UV-vis spectroscopy and clearly highlighted that AuNP aggregation, and hence gold MeLLD formation, does not occur in the absence of TTF in DCE (Figure 2C,D; and Figure S5 in the Supporting Information SI-5B). Third, visual inspection of unshaken (or unsonicated) biphasic reaction systems, prepared as described in Figure $1 \mathrm{~A}$, indicate that gold MeLLD formation only occurs with emulsification (see Figure S6 in the Supporting Information SI-5C, discussed vide infra).

Investigating the Conductivity of Gold MeLLDs. The possibility of metallic (electronic) conductivity on the macroscale for gold MeLLDs, formed with both colloidal AuNP solutions, was investigated by electrochemical impedance spectroscopy (EIS, see Figure 3). The equivalence electric circuit diagram of the impedance data and a scheme outlining the interpretation of each element are also presented in Figure 3. $R_{1}$ represents the solution resistance (i.e., the resistance of the solution to ionic conductivity). The values of $R_{1}$ for pure water and DCE containing $1 \mathrm{mM}$ TTF were in agreement, with the expected lower conductivities of the organic solvent. Additionally, the resistance of the water/DCE interface is lower than the resistance of pure water, as expected for the presence of two resistors in a parallel circuit (see Table S5 in the Supporting Information SI-5D). The value of $R_{1}$ is smaller for the $14 \mathrm{~nm}$ AuNP gold MeLLDs due to the better packing possible with smaller NPs. Thus, $\mathrm{R}_{1}$ for a gold MeLLD is the sum of the individual resistances of the inter-AuNP regions, likely to consist of a TTF-matrix as discussed vide supra. On polarizing the electrodes (with an applied potential difference of $250 \mathrm{mV}$ ), it follows that each AuNP is effectively bipolar in nature due to the applied electric field between the two Pt electrodes. Each bipolar AuNP has a corresponding double-layer of ions surrounding it that gives rise to a pseudocapacitance represented by the $\mathrm{CPE}_{1}$ element. This element may also include a contribution from the capacitance of the Pt wires as the CPE will be dominated by the smallest capacitance, i.e., that of individual AuNPs. $R_{2}$ represents a polarization resistance of the interface. The key finding from Figure 3 is that the impedance spectra excludes the possibility of metallic (electronic) conductivity of the gold MeLLDs on the macroscale since the real components of the complex impedance are very high at lower frequencies. Thus, under an applied electric field the interfacial liquid-like gold film effectively reduces the distance that ions much conduct between the two Pt electrodes, with "islands" of AuNPs in between that are bipolar in nature.

Gold MeLLD Formation Mechanism. (i). Electrochemical Charge Transfer and Electrostatic Interactions between Lipophilic Tetrathiafulvalene and Hydrophilic Colloidal AuNPs. Previous surface enhanced Raman spectroscopy (SERS) studies have highlighted the ability of neutral TTF to competitively displace adsorbed citrate efficiently from the surface of gold substrates (including aggregated colloidal AuNPs). ${ }^{39,40}$ TTF was found to be present as its radical cation, $\mathrm{TTF}^{\cdot+}$, when adsorbed on gold, and thus, by inference, a charge transfer reaction occurred whereby adsorbed neutral TTF injected an electron into the gold substrate. ${ }^{39,40}$ An alternative and equally plausible scenario is the oxidation of TTF by the AuNP followed by the subsequent electrostatic adsorption of positively charged $\pi^{\circ}{ }^{*}$ to the negatively charged AuNP. Although not observed by SERS in previous reports for TTF adsorbed on bulk gold or AuNP aggregates, the presence of the dication, $\mathrm{TF}^{2+}$, at the surface of the AuNPs cannot be excluded. In our system (Figure $1 \mathrm{~A}$ ), immediately after placing the aqueous and DCE phases in contact, neutral TTF molecules partition between the oil and water phases, with subnanomolar concentrations of TTF partitioning to water (see Supporting Information SI-6A). Thus, charge transfer, and the ensuing displacement of citrate with the adsorbed radical cation (i.e., $T \mathrm{TF}_{\mathrm{ads}}^{\cdot+}$ ), may occur both homogeneously in bulk water and heterogeneously at the interface (see Figure S7 and eqs S11 to S14 in the Supporting Information SI-6B). 


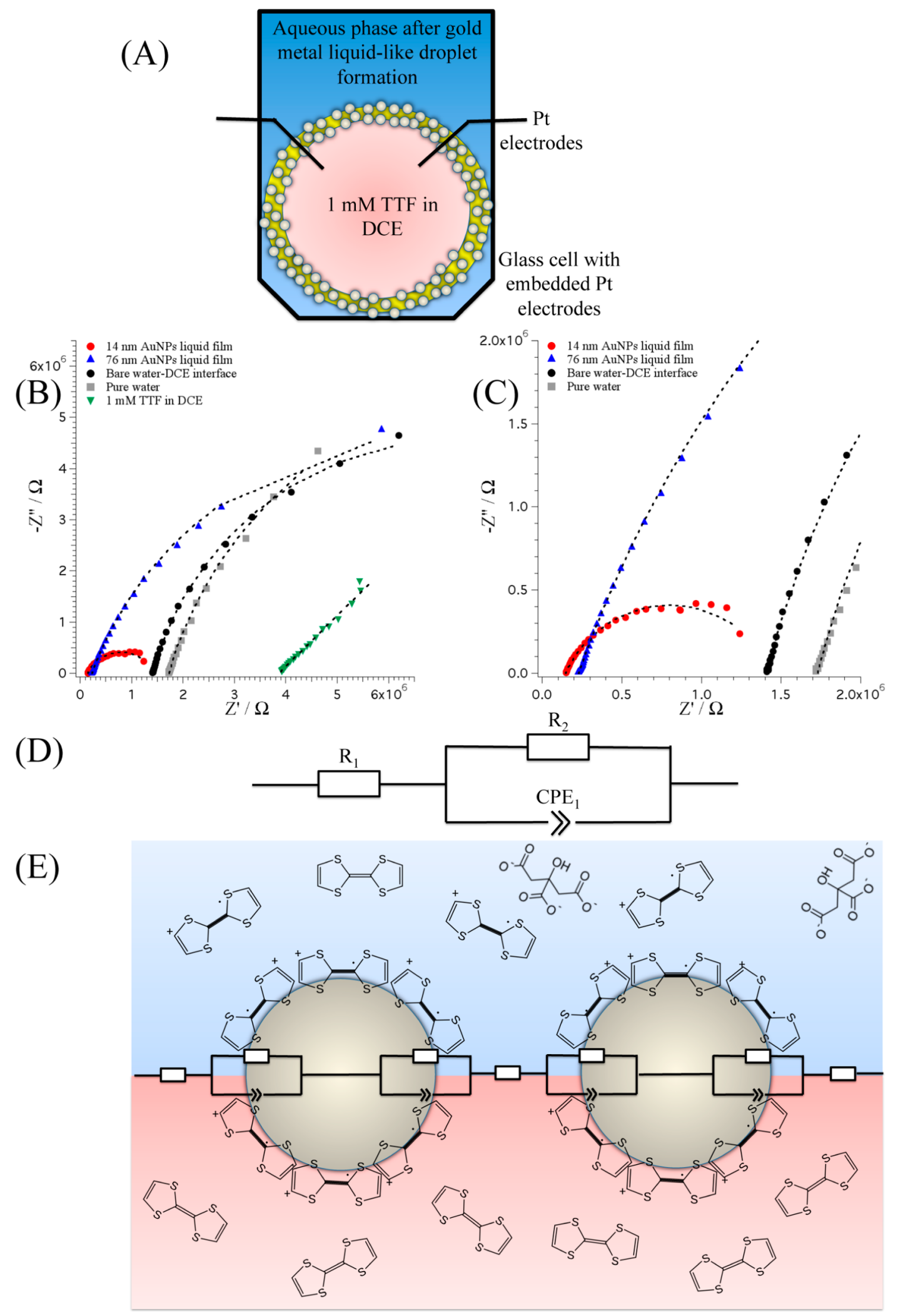

Figure 3. Electrochemical impedance spectroscopy (EIS) of gold MeLLDs. (A) Schematic of the glass cell with embedded Pt electrodes used to carry out the EIS measurements with solutions of $1 \mathrm{mM}$ TTF in DCE, pure water, the bare water-DCE (containing $1 \mathrm{mM}$ TTF) interface, and the water-DCE (containing $1 \mathrm{mM}$ TTF) interface covered in AuNP multilayers of either the smaller $\left(x=14, y=5, z=2.67 \times 10^{12}\right.$, see Figure $\left.1 A\right)$ or larger $\left(x=76, y=8, z=1.35 \times 10^{10}\right.$, see Figure 1A) AuNPs. (B) Nyquist plots for each cell and (C) magnification of the plots in (B). (D) The reduced equivalence electric circuit diagram of the impedance data and (E) a schematic of the TTF $F_{\text {ads }}^{-+}$-coated AuNPs at the water-DCE soft interface with an overlay of the impedance equivalence model used to simulate the EIS responses. 
The thermodynamic driving force underpinning the charge transfer process may be readily understood on the basis of the relative magnitudes of the reduction potentials of TTF in water $(\mathrm{w})\left(\left[E_{T T F \cdot+} / T_{T F}^{0}\right]_{A V S}^{\mathrm{w}}=4.14 \mathrm{~V}\right)$ and TTF in DCE (oil) $\left(\left[E_{T T F \cdot+} / T_{T F}^{0}\right]_{\text {AVS }}^{\text {oil }}=5.00 \mathrm{~V}\right)$, and the work function of the AuNPs $\left(\phi_{\text {AuNP }}=5.32 \mathrm{~V}\right)$, here expressed on the absolute vacuum scale (AVS, see Figure S8 in the Supporting Information SI-6C) Thus, although a substantially greater thermodynamic driving force exists for the homogeneous over the heterogeneous aggregation route, the two processes are competitive due to the low aqueous solubility of TTF. Irrespective of the path followed, in both instances, electron transfer processes occur until the system reaches Fermi-level equilibration, in which $\phi_{\text {AuNP }}$ is raised to a more reducing potential (Figure 4). ${ }^{41}$ Fermi-level equilibration may also influence the adsorption/desorption dynamics of citrate and TTF species. After equilibrium, the more reduced AuNPs may induce the removal of anionic citrate ligands electrostatically, further facilitating the absorption of $\mathrm{TT}^{{ }^{*+}}$. The latter inference is supported by the observations of Weitz et al., who noted that tetracyanomethanediquinone (TCNQ), adsorbed as its radical anion, $\mathrm{TCNQ}^{\bullet-}$, cannot displace citrate. ${ }^{40}$ As an electron acceptor, TCNQ oxidizes the surface of the AuNP during charge transfer, lowering $\phi_{\text {AuNP }}$ to a less reducing potential, thereby increasing the electrostatic attraction between citrate and the surface of the AuNPs.

(ii). The Role of Emulsifying the Biphasic System. Vigorous shaking of the reaction cell, emulsifying the water and oil phases, significantly increases the rate of citrate displacement by $\mathrm{TTF}_{\mathrm{ads}}^{\circ}$, and therefore in effect the rate of aggregation of the AuNPs, by increasing the surface area of the oil droplets in contact with water. The aggregation of the AuNPs on displacement of citrate by $\mathrm{TF}_{\mathrm{ads}}^{\circ+}$ may be explained using DerjaguinLandau-Verwey-Overbeck (DLVO) theory. ${ }^{2}$ The surface charge densities of $\mathrm{TTF}_{\mathrm{ads}}^{\circ-}$-coated AuNPs are considerably lower than with citrate ligands (as evidenced by zeta $(\zeta)$-potential measurements, see Table S2 in the Supporting Information SI-3) such that Coulombic repulsions are no longer a sufficient barrier to keep the AuNP cores separated at distances outside the sphere of influence of the van der Waals (vdWs) forces.

SERS studies by Sandroff et al. $^{39}$ indicate that $\mathrm{TF}_{\text {ads }}^{\bullet+}$ maintains its aromaticity on AuNPs. Briefly, $\mathrm{TT}_{\mathrm{ads}}^{\circ+}$ lies flat on the surface of gold, and no covalent bonds are formed between gold and $\mathrm{TF}_{\mathrm{ads}}^{\circ}$, primarily due to the low nucleophilicity of the sulfur atoms in TTF. ${ }^{42}$ This is crucial as, in the absence of covalent bond formation, $T_{\mathrm{T}}^{\circ} \cdot+$ ds does not dissociate and maintains its aromaticity. Indeed, the strong adsorption of $T \mathrm{~F}_{\mathrm{ads}}^{\cdot+}$ arises from an electrostatic attraction between the delocalized charge of $T \mathrm{~F}_{\mathrm{ads}}^{\cdot+}$ and the surface of the gold. By maintaining its aromaticity $\mathrm{TF}_{\mathrm{ads}}^{\circ+}$ may partake in further interactions that counteract the Coulombic

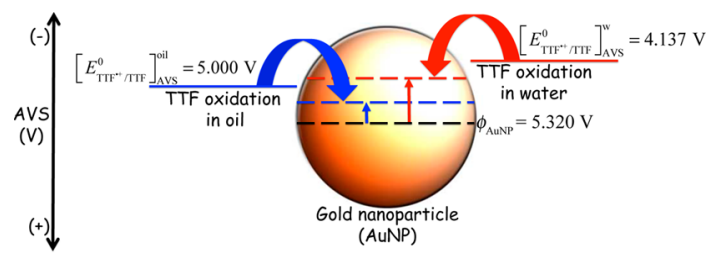

Figure 4. Schematic of the shift in the work function of the AuNPs during charge (electron) transfer with TTF to more reducing potentials.

repulsions such as $\pi-\pi$ interactions with neighboring $\mathrm{TTF}_{\text {ads }}^{\bullet+}$ molecules. The latter and other interactions (for example, nonbonding $\mathrm{S}-\mathrm{S}$ interactions) play an important role in the AuNP aggregation and gold MeLLD formation processes (see Supporting Information SI-6D). Additionally, vigorous shaking, or alternatively ultrasonication, effectively prevents the formation of larger aggregates of AuNPs by rapidly facilitating the spontaneous adsorption of individual, small aggregates of $\mathrm{TF}_{\mathrm{ads}}^{-+}$-coated AuNPs at the interface. The driving force behind this spontaneous interfacial adsorption of AuNPs is the diminution of excess surface energy at an early stage of the AuNP aggregation process induced by TTF. ${ }^{43,44}$ In accordance with the latter, control experiments where the biphasic system was left to sit unshaken failed to produce metallic AuNP films (see Figure S6 in the Supporting Information SI-5C). Instead, large black AuNP aggregates formed which precipitated with time both onto the interface and the bottom of the reaction vial. A schematic of the full MeLLD formation process is presented in Figure 5.

The Surprising Maintenance of Reflectivity and Mechanical Properties of Multilayer Gold MeLLDs. Typically during MeLLF formation the water-oil or water-air interfacial surface tension $(\gamma)$ steadily decreases as the number of AuNPs adsorbed at the interface $(N)$ increases up to the moment of interfacial crumpling. ${ }^{45-47}$ If the interface does buckle then diffuse scattering dominates due to the corrugated interfacial surface. A resultant increase in absorption of the incident light would give the interface a black appearance, while the metallic luster of the film would be lost. Clearly, herein, for MeLLD formation an alternative mechanism prevails, outlined in Figure 5 , as indicated by (i) the absence of irreversible buckling or fracture, with the maintenance of spectral reflectivity, at very high surface loadings of AuNPs far in excess of monolayer conditions (Figure 1F) and (ii) the complete absence of "expelled" AuNPs from the interface after gold MeLLD formation (as discussed vide supra, see Figure S4 in the Supporting Information SI-5A).

The contact angles $\left(\theta_{c}\right)$ between (i) an unmodified droplet of DCE (containing $1 \mathrm{mM}$ TTF) and (ii) gold MeLLDs of varying AuNP surface coverage (0.5, 1, and 3 monolayers, respectively) and a glass surface, indicative of increasing or decreasing $\gamma$, were determined by analyzing the shape of a sessile droplet on a quartz 

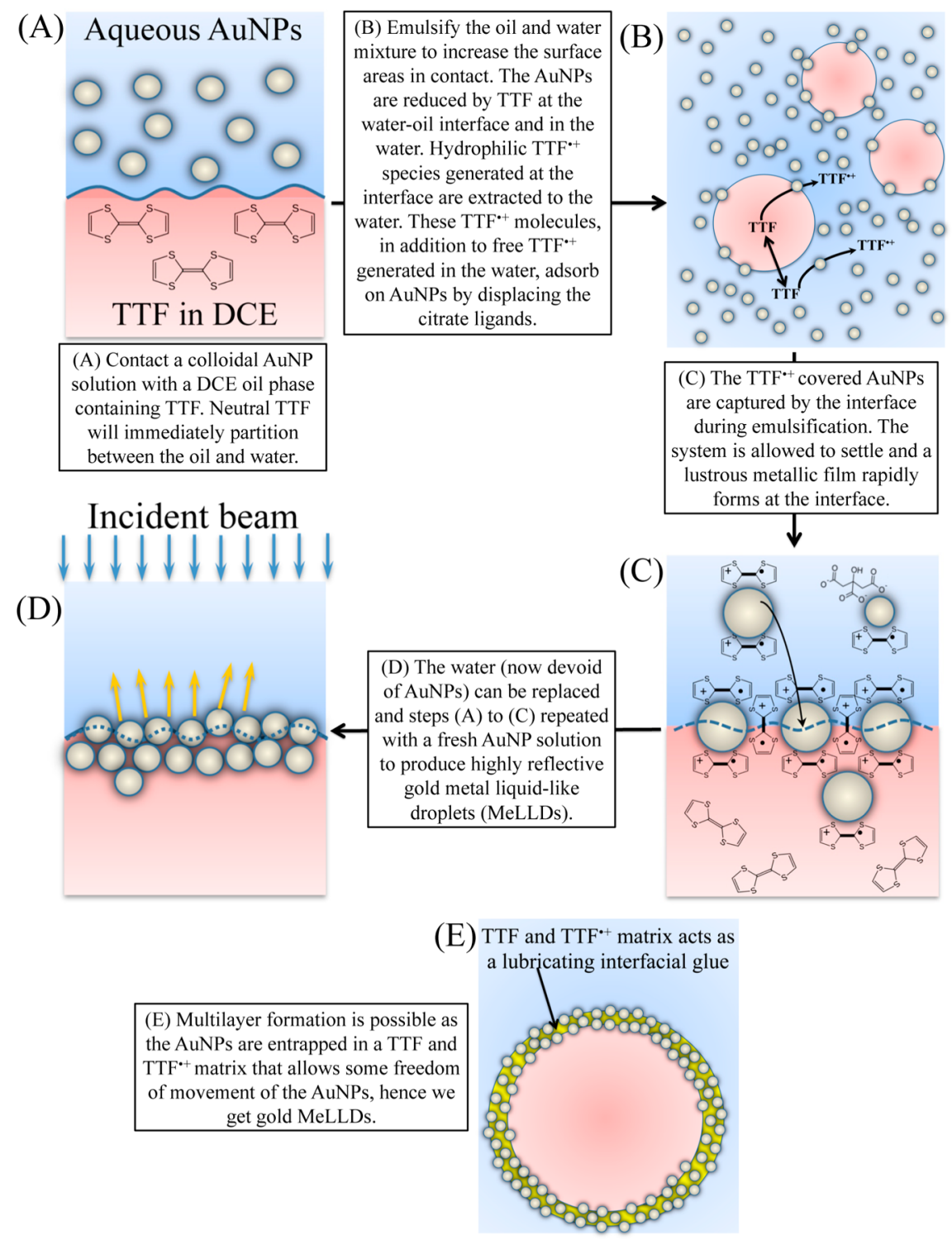

Figure 5. Schematic of the gold MeLLD formation process. The homogeneous and heterogeneous charge transfer reactions between TTF and AuNPs in (B) are described in detail in Figure S7 (see Supporting Information SI-6B).

substrate in the presence of an aqueous solution (see Figure S9 in the Supporting Information SI-7A)). ${ }^{48,49} \mathrm{~A}$ slight $2^{\circ}$ reduction in $\theta_{c}$ was observed for a gold MeLLD with half a monolayer of AuNPs adsorbed compared to a bare droplet. For single monolayer and multilayer (i.e., equivalent to 3 monolayers) surface coverage, however, $\theta_{c}$ increased slightly by $4^{\circ}$ compared to the bare droplet, indicating of a net increase in $\gamma$. Initially, this might seem counterintuitive as interfacial AuNP adsorption processes typically decrease excess surface energy, thus reducing $\gamma$ as mentioned. ${ }^{43,44}$ Herein, we believe that the surface tension increases due to the interfacial accumulation of extremely hydrophilic $\mathrm{TTF}^{\circ+}$ in the interfacial matrix of the AuNP film (Figure 5E). As a result, the net change of the energy per unit area of interface will increase substantially compared to that of the bare water-oil interface resulting in an increased surface tension. However, film formation is still thermodynamically favorable since the global interfacial excess surface energy in the system is lowered as the total surface area of exposed AuNPs is reduced during gold MeLLD formation.

Multilayer formation while maintaining spectral

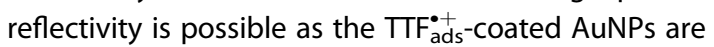
mobile within this matrix. The relatively low charge density of $\mathrm{TT}_{\mathrm{ads}}^{\cdot+}$-coated AuNPs means a close-packed AuNP assembly is favored in the matrix. Considerable additional dispersive and attractive forces, of greater complexity than DLVO theory for bulk AuNP interactions, control AuNP interactions within interfacial fluid films (see Supporting Information SI-6E). ${ }^{21}$ The precise balance of these interparticle forces for our system allows the interfacial matrix of neutral $T \mathrm{TF}, \mathrm{TT}_{\mathrm{ads}}^{-+}$and free $\pi^{\bullet+}$ to act as lubricating molecular glue, binding the AuNPs together, while retaining some freedom of 
movement to facilitate multilayer formation. In contrast, as noted, the interface buckles when monolayer surface coverage's are exceeded for the majority of continuous reflective liquid mirrors reported to date. ${ }^{50}$

A dramatic illustration of the liquid-like flexibility of a gold MeLLD is the reversible deformation of the gold film upon compression and decompression (see Movie S5 in the Supporting Information SI-1). Compression is achieved by simply reducing the volume of the droplet by withdrawing the inner DCE phase with a micropipette, while subsequent expansion of the droplet by reinserting DCE decompresses the gold film. As the droplet size becomes progressively smaller, the interfacial gold film becomes compressed to the limits of its stability in two-dimensions and thus "explores" the third dimension as the film deforms. ${ }^{51}$ During film deformation, protrusions or wrinkles extend several microns into the aqueous phase (as clearly seen in Movie S5 in the Supporting Information SI-1). The wrinkles coexist with areas of undeformed, flat film and a further reduction in droplet volume (i.e., increasing compression) causes an increase in the fraction of the gold film wrinkled. Thus, unlike the situation for the formation of spectrally reflective multilayer gold MeLLDs described thus far, upon compression spectral reflectivity is significantly diminished. The latter is a kinetic effect arising from the limited mobility of the AuNPs within the interfacial lubricating layer preventing their rearrangement on the short time scale involved. However, as the volume of the droplet is increased, the protrusions flatten, are reincorporated into the flat gold film and allow full restoration of the gold MeLLDs metallic luster upon decompression (see Movie S5 in the Supporting Information SI-1).

Herein, the mechanism of film collapse upon compression is different to those typically observed, i.e., the fracture or solubilization processes that underpin the mechanisms of buckling for monolayers of lipids or NPs at liquid-liquid or liquid-air interfaces. ${ }^{51}$ During fracture well-ordered or rigid monolayer films collapse upon compression causing irreversible loss of material to the bulk phases or the formation of multilayered aggregates at the air side of the interface. ${ }^{51}$ During solubilization highly fluidic films eject material out of the interface into the bulk phase upon compression. ${ }^{52}$ Thus, neither mechanism allows reversible deformation of the films upon decompression in contrast to the reversibility shown by gold MeLLDs. As noted, $\pi^{\circ} F_{\text {ads }}^{\circ+}$ coated AuNPs are encased in a lubricating interfacial

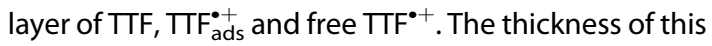
film is on the nanoscale, depending on the size of the AuNPs in the film and number of monolayers adsorbed. Therefore, the film is flexible enough to form protrusions and buckle upon compression, but cohesive enough to reform a smooth spectrally reflective surface on decompression. The cohesive nature of the film allows it to act as a glue preventing the "solubilization" or irreversible expulsion of AuNPs from the interface into the bulk phases upon compression. Finally, the AuNPs are stabilized in the film by a balance of interparticle forces, as discussed in Supporting Information $\mathrm{SI}-6 \mathrm{E}$, preventing their irreversible aggregation. The reversibility of film deformation in a MeLLD type system is unusual and, indeed, such reversible deformation of monolayer films in general, be they purely lipid-based ${ }^{51}$ or consisting of $\mathrm{NPs}^{53}$ is rare. Schultz et al. prepared monolayers of dodecanethiolligated AuNPs suspended in heptane using a Langmuirtrough. ${ }^{53}$ Reversibility of deformation upon decompression was noted when excess dodecanethiol was added to the solution. Reminiscent of the interparticle interactions in the lubricating interfacial layer discussed herein, the latter reversibility was attributed to a tunable ligand-induced steric repulsion between AuNPs in the presence of excess thiol, which in turn influenced the vdWs attraction between AuNPs in the film and prevented their irreversible aggregation during compression.

A final experiment was performed to highlight that the protrusions or wrinkles in the gold MeLLD, formed upon compression on withdrawing of DCE from the droplet, are not permanent and that a substantial amount of the luster of the gold MeLLD may be restored without expanding the volume of the DCE droplet (see Figure S10 in the Supporting Information SI-7B). Half of the DCE was removed from a gold MeLLD and discarded permanently. The resulting film formed on the smaller droplet was wrinkled and subjected to two treatments (i) vigorous agitation using a vortex shaker and (ii) ultrasonication. During both treatments the gold MeLLDs were broken apart and reassembled to form multilayer gold MeLLDs upon settling. In the case of vortex shaking little of the metallic luster was restored and considerable wrinkling remained. In contrast, ultrasonication substantially restored the luster of the gold MeLLD. The difference is attributed to the size differences in the microdroplets formed during both treatments. Clearly, during ultrasonication much smaller droplets were formed ( $<10 \mu \mathrm{m}$ in size) and thus all wrinkles greater than this size were permanently destroyed. However, with vortex shaking the large micron size wrinkles remain as the microdroplets formed were even larger (tens of microns in some instances).

The dynamics of reversible gold MeLLD wrinkling are expected to provide fertile ground for future research that may deliver valuable insights into the fundamental physics underlying the collapse and folding of biological membranes and cellular structures, ${ }^{54}$ for example the inner surface of lungs. ${ }^{55}$

Finally, the gold MeLLDs are kinetically stable. If the DCE within the droplet is replenished at regular intervals, to replace that lost by evaporation at room temperature, no obvious loss in luster or spectral reflectivity is observed for over a year. 


\section{CONCLUSIONS}

Gold metal liquid-like droplets (MeLLDs) were formed by the biphasic reaction of a lipophilic electron donor, tetrathiafulvalene, and hydrophilic citratestabilized gold nanoparticles. These MeLLDs were able to retain their reflectivity at surface coverages in excess of monolayer conditions but were not conductive on the macroscale. The MeLLDs were structurally robust, capable of being disrupted and reforming instantaneously ad infinitum. The MeLLDs are reversibly deformable upon compression and decompression (i.e., in withdrawing and reinjecting the oil phase) and kinetically stable for extended periods of time. We believe this strategy of forming noncovalent, lubricating, interfacial glue layers is generic and are currently investigating other ligands with added functionality (such as the ability to be cross-linked) along with alternative solvent combinations. Future studies will involve determining the microscale conductivity of these films by scanning electrochemical microscopy (SECM), further in-depth studies of the mechanical properties of the MeLLDs, and carrying out SERS studies of the gold MeLLDs in situ (i.e., without their transfer to solid substrates). The utility of these films is immense, with immediate applications envisioned in optics (as filters and mirrors as shown herein), biomedical research (size-selective membranes for dialysis, or drug-delivery capsules), model systems to probe the collapse and folding of biological membranes and cellular structures, sensors (SERS at fluid interfaces), catalysis (nanoscale bioreactors) and perhaps as an alternative gold recovery method in the mining industry.

\section{EXPERIMENTAL SECTION}

Reagents. All chemicals were used as received without further purification. All aqueous solutions were prepared with ultrapure water (Millipore Milli-Q, specific resistivity $18.2 \mathrm{M} \Omega \cdot \mathrm{cm}$ ). The solvents used were 1,2-dichloroethane (DCE, $\geq 99.8 \%$, Fluka) and acetonitrile ( $\geq 99.5 \%$, Riedel-de-Haen). Hydrogen tetrachloroaurate(III) hydrate $\left(\mathrm{HAuCl}_{4} \cdot 3 \mathrm{H}_{2} \mathrm{O}, 99.999 \%, 49 \% \mathrm{Au}\right)$ was provided by Alfa Aesar. Anhydrous trisodium citrate (98\%) and tetrathiafulvalene ( $T \mathrm{TF}, \geq 99 \%$ ) were purchased from Acros.

Colloidal AuNP Preparation and Characterization. Two solutions of colloidal AuNPs were prepared by the reduction of $\mathrm{HAuCl}_{4} \cdot 3 \mathrm{H}_{2} \mathrm{O}$ in water by trisodium citrate, where the goldto-sodium citrate (gold/stabilizer) mole ratio was varied to produce AuNPs of the desired size, as reported by Frens. ${ }^{56}$ Briefly, $41.5 \mathrm{mg}$ of $\mathrm{HAuCl}_{4} \cdot 3 \mathrm{H}_{2} \mathrm{O}$ was dissolved in $300 \mathrm{~mL}$ of deionized water and heated to $100{ }^{\circ} \mathrm{C}$ in a round-bottomed flask, with stirring and under reflux to maintain a constant volume. The desired quantity of a trisodium citrate solution was rapidly injected into the round-bottomed flask upon commencement of boiling. Specifically, $9 \mathrm{~mL}$ of a $1 \% \mathrm{w} / \mathrm{v}$ trisodium citrate solution was injected to prepare the smaller $14 \mathrm{~nm}$ AuNPs and $2.1 \mathrm{~mL}$ to prepare the larger $76 \mathrm{~nm}$ AuNPs. After approximately $30 \mathrm{~s}$ the solution initially turned dark black, before changing to red for the smaller AuNPs and purple for the larger AuNPs. The solution was maintained at its boiling point for $45 \mathrm{~min}$ and subsequently cooled down to generate a stable colloidal suspension.

The morphologies of both colloidal AuNP solutions were characterized by scanning electron microscopy (SEM) and transmission electron microscopy (TEM; see Supporting Information SI-2). An alternative method of determining the mean diameters and approximate concentrations of the colloidal AuNP solutions was also carried out by UV-vis spectroscopy, as detailed by Haiss et al. ${ }^{57}$ (see Supporting Information SI-3). UV-vis spectra were obtained using an Ocean Optics HR2000+ high-resolution miniature fiber optic spectrophotometer coupled with a DH-2000-BAL deuterium tungsten halogen light source. The optical path length was $1 \mathrm{~cm}$. From the latter method, the mean diameters of the smaller and larger AuNPs were 14 and $76 \mathrm{~nm}$, respectively.

Zeta $(\zeta)$-potential and dynamic light scattering (DLS) measurements were carried out on a Nano ZS Zetasizer (Malvern Instruments, U.K.), with irradiation $(\lambda=633 \mathrm{~nm}$ ) from a $\mathrm{He}-\mathrm{Ne}$ laser, using Dispersion Technology Software (DTS). Measurements were obtained for the as prepared, aggregate-free, citrate coated AuNP solutions and, subsequently, aggregated $T_{T} F_{\text {ads }}^{*}$-coated AuNP samples prepared by very lightly shaking the AuNP colloidal solution with a drop of DCE containing $1 \mathrm{mM}$ TTF for $10 \mathrm{~s}$ (see Table S4 in Supporting Information SI-2).
The AuNP samples (approximately $0.75 \mathrm{~mL}$ ) were injected into a folded capillary cell. The $\zeta$-potential $(\mathrm{mV})$ was elucidated from the measured electrophoretic mobility using the Smoluchowski approximation $^{58,59}$ of Henry's equation. DLS measures Brownian motion and relates the particles speed to its size using the Stokes-Einstein equation. The particles were illuminated with the laser and the intensity fluctuations of the scattered light analyzed. All particle size measurements were carried out at $25^{\circ} \mathrm{C}$ and a 2 min equilibration time employed.

Gold Metal Liquid-Like Droplets (MeLLDs) Preparation and Characterization. Our approach to preparing MeLLDs is facile and rapid, with the entire process taking from 60 to $300 \mathrm{~s}$ to complete depending on the size of the AuNPs (see Figure 5 and Movies S1 to S3 in the Supporting Information SI-1). The procedure involves (A) contacting aliquots of aqueous citrate-stabilized AuNPs with an oil droplet of 1,2-dichloroethane (DCE) that contains $1 \mathrm{mM}$ tetrathiafulvalene (TTF), (B) vigorously shaking the two immiscible solutions for from 30 to $240 \mathrm{~s}$ (longer time required for the larger AuNPs), (C) allowing the oil and water to separate revealing a clear aqueous phase and an oil droplet resembling "liquid gold", and, finally, (D) replacing the water phase with a fresh AuNP solution and repeating steps (A) to (C) multiple times to form films of the desired thickness and reflectivity. The morphologies and packing arrangement of the gold MeLLDs formed with the smaller and larger AuNPs were investigated both by TEM and SEM. The coatings of AuNPs around the oil droplet were transferred to either standard carbon-coated copper grids (200-mesh, for TEM analysis) or a silicon substrate (for SEM analysis) by carefully touching the surface of the AuNP-coated macroscopic droplets (see Supporting Information SI-2). The interfacial surface coverages were estimated from the approximate concentrations of the colloidal AuNP solutions, determined by UV-vis spectroscopy (see Supporting Information SI-3), using the procedure detailed in the Supporting Information SI-3.

Kinetic experiments monitoring gold MeLLD formation were performed by UV-vis spectroscopy using an Ocean Optics HR2000+ high-resolution miniature fiber optic spectrophotometer coupled with a DH-2000-BAL deuterium tungsten halogen light source and controlled by a custom LabView program. The LabView program recorded spectra at regular 5 min intervals, typically over several hours. As described in Figure 1A, $1 \mathrm{~mL}$ of the DCE organic phase containing TTF was contacted with $1.5 \mathrm{~mL}$ of the aqueous AuNP colloidal solution in a quartz cuvette with a Teflon cap to prevent evaporation. The volumes of the aqueous and organic phases were chosen so that the spectra of the aqueous phase were obtained slightly above the interfacial region. The kinetic experiments were carried out under quiescent conditions in air. 
The simple experimental setup used to test the conductivity of the gold MeLLDs, formed using both 14 and $76 \mathrm{~nm}$ AuNPs as described vide supra, by electrochemical impedance spectroscopy (EIS) is shown schematically in Figure 3A. The EIS measurements were recorded in a 2-electrode setup using a Metrohm Autolab PGSTAT 12 with FRA V.4.9 software, within the frequency range of 0.01 to $1000 \mathrm{~Hz}$, with an applied potential difference of $250 \mathrm{mV}$ between both Pt electrodes and with the amplitude of the sinusoidal (AC) perturbation set at $10 \mathrm{mV}$. The experimental EIS data were fit to an appropriate equivalent circuit using ZSimpWin software (Princeton Applied Research).

The contact angles $\left(\theta_{c}\right)$ of a DCE droplet containing $1 \mathrm{mM}$ TTF and various gold MeLLDs of differing surface coverage (0.5, 1 , and 3 monolayers of AuNPs), indicative of the water-oil interfacial surface tension $(\gamma)$, were measured using the sessile drop method. Briefly, each droplet was placed in a large quartz cell filled with water. The glassware was previously cleaned with aqua regia and $\mathrm{O}_{2}$ plasma. The wetting of the glass by each droplet was determined using a Drop Shape Analyzer DSA100 instrument (Krüss, Germany) and $\theta_{c}$ between the droplet and the glass surface measured.

Conflict of Interest: The authors declare no competing financial interest.

Acknowledgment. Véronique Amstutz (EPFL) is thanked for microscopy characterizations of the colloidal gold nanoparticles and gold metal liquid-like droplets. This work was supported by the Swiss Science Foundation through the NCCR "MUST" program.

Supporting Information Available: Supporting movies, characterization by transmission electron microscopy, scanning electron microscopy, UV/spectroscopy, zeta ( () -potential and dynamic light scattering (DLS) measurements, estimation of the interfacial coverage, control experiments and supporting notes on the mechanism of metal liquid-like droplets formation. This material is available free of charge via the Internet at http:// pubs.acs.org.

\section{REFERENCES AND NOTES}

1. Sönnichsen, C.; Reinhard, B. M.; Liphard, J.; Alivisatos, A. P. A Molecular Ruler Based on Plasmon Coupling of Single Gold and Silver Nanoparticles. Nat. Biotechnol. 2005, 23, 741-745.

2. Ghosh, S. K.; Pal, T. Interparticle Coupling Effect on the Surface Plasmon Resonance of Gold Nanoparticles: From Theory to Applications. Chem. Rev. 2007, 107, 4797-4862.

3. Es-Souni, M.; Fischer-Brandies, H.; Es-Souni, M. Versatile Nanocomposite Coatings with Tunable Cell Adhesion and Bactericidity. Adv. Funct. Mater. 2008, 18, 3179-3188.

4. Puntes, V. F.; Krishnan, K. M.; Alivisatos, A. P. Colloidal Nanocrystal Shape and Size Control: The Case of Cobalt. Science 2001, 291, 2115-2117.

5. Borra, E. F.; Seddiki, O.; Angel, R.; Eisenstein, D.; Hickson, P.; Seddon, K. R.; Worden, S.P. Deposition of Metal Films on an Ionic Liquid as a Basis for a Lunar Telescope. Nature 2007, 447, 979-981.

6. Khan, Z.; Kumar, R.; Mohammed, W.; Hornyak, G.; Dutta, J. Optical Thin Film Filters of Colloidal Gold and Silica Nanoparticles prepared by a Layer-by-Layer Self-Assembly Method. J. Mater. Sci. 2011, 46, 6877-6882.

7. Saha, K.; Agasti, S. S.; Kim, C.; Li, X.; Rotello, V. M. Gold Nanoparticles in Chemical and Biological Sensing. Chem. Rev. 2012, 112, 2739-2779.

8. Daniel, M.-C.; Astruc, D. Gold Nanoparticles: Assembly, Supramolecular Chemistry, Quantum-Size-Related Properties, and Applications toward Biology, Catalysis, and Nanotechnology. Chem. Rev. 2004, 104, 293-346.

9. Borisova, D.; Möhwald, H.; Shchukin, D. G. Mesoporous Silica Nanoparticles for Active Corrosion Protection. ACS Nano 2011, 5, 1939-1946.

10. Zhang, X.-T.; Sato, O.; Taguchi, M.; Einaga, Y.; Murakami, T.; Fujishima, A. Self-Cleaning Particle Coating with Antireflection Properties. Chem. Mater. 2005, 17, 696-700.
11. Kowalczyk, B.; Lagzi, l.; Grzybowski, B. A. "Nanoarmoured" Droplets of Different Shapes Formed by Interfacial Self-Assembly and Crosslinking of Metal Nanoparticles. Nanoscale 2010, 2, 2366-2369.

12. Atwater, H. A.; Polman, A. Plasmonics for Improved Photovoltaic Devices. Nat. Mater. 2010, 9, 205-213.

13. Huda, S.; Smoukov, S. K.; Nakanishi, H.; Kowalczyk, B.; Bishop, K.; Grzybowski, B. A. Antibacterial Nanoparticle Monolayers Prepared on Chemically Inert Surfaces by Cooperative Electrostatic Adsorption (CELA). ACS Appl. Mater. Interfaces 2010, 2, 1206-1210.

14. Binder, W. H. Supramolecular Assembly of Nanoparticles at Liquid-Liquid Interfaces. Angew. Chem., Int. Ed. 2005, 44, 5172-5175.

15. Edel, J. B.; Kornyshev, A. A.; Urbakh, M. Self-Assembly of Nanoparticle Arrays for Use as Mirrors, Sensors, and Antennas. ACS Nano 2013, 7, 9526-9532.

16. Wang, D.; Duan, H.; Mohwald, H. The Water/Oil Interface: the Emerging Horizon for Self-Assembly of Nanoparticles. Soft Matter 2005, 1, 412-416.

17. Boker, A.; He, J.; Emrick, T.; Russell, T. P. Self-Assembly of Nanoparticles at Interfaces. Soft Matter 2007, 3, 12311248.

18. Yogev, D.; Efrima, S. Novel Silver Metal Liquidlike Films. J. Phys. Chem. 1988, 92, 5754-5760.

19. Reincke, F.; Hickey, S. G.; Kegel, W. K.; Vanmaekelbergh, D. Spontaneous Assembly of a Monolayer of Charged Gold Nanocrystals at the Water/Oil Interface. Angew. Chem., Int. Ed. 2004, 43, 458-462.

20. Park, Y.-K.; Yoo, S.-H.; Park, S. Assembly of Highly Ordered Nanoparticle Monolayers at a Water/Hexane Interface. Langmuir 2007, 23, 10505-10510.

21. Turek, V. A.; Cecchini, M. P.; Paget, J.; Kucernak, A. R.; Kornyshev, A. A.; Edel, J. B. Plasmonic Ruler at the LiquidLiquid Interface. ACS Nano 2012, 6, 7789-7799.

22. Konrad, M. P.; Doherty, A. P.; Bell, S. E. J. Stable and Uniform SERS Signals from Self-Assembled Two-Dimensional Interfacial Arrays of Optically Coupled Ag Nanoparticles. Anal. Chem. 2013, 85, 6783-6789.

23. Lee, K. Y.; Cheong, G.-W.; Han, S. W. C 60 -Mediated SelfAssembly of Gold Nanoparticles at the Liquid/Liquid Interface. Colloids Surf., A 2006, 275, 79-82.

24. Lee, K. Y.; Kim, M.; Hahn, J.; Suh, J. S.; Lee, I.; Kim, K.; Han, S. W. Assembly of Metal Nanoparticle-Carbon Nanotube Composite Materials at the Liquid/Liquid Interface. Langmuir 2006, 22, 1817-1821.

25. Kim, K.; Han, H. S.; Choi, I.; Lee, C.; Hong, S.; Suh, S.-H.; Lee, L. P.; Kang, T. Interfacial Liquid-State Surface-Enhanced Raman Spectroscopy. Nat. Commun. 2013, 4, 218210.1038/ ncomms3182.

26. Fang, P.-P.; Chen, S.; Deng, H.; Scanlon, M. D.; Gumy, F.; Lee, H. J.; Momotenko, D.; Amstutz, V.; Cortés-Salazar, F.; Pereira; et al. Conductive Gold Nanoparticle Mirrors at Liquid/Liquid Interfaces. ACS Nano 2013, 7, 9241-9248.

27. Du, K.; Knutson, C. R.; Glogowski, E.; McCarthy, K. D.; Shenhar, R.; Rotello, V. M.; Tuominen, M. T.; Emrick, T.; Russell, T. P.; Dinsmore, A. D. Self-Assembled Electrical Contact to Nanoparticles Using Metallic Droplets. Small 2009, 5, 1974-1977.

28. Du, K.; Glogowski, E.; Tuominen, M. T.; Emrick, T.; Russell, T. P.; Dinsmore, A. D. Self-Assembly of Gold Nanoparticles on Gallium Droplets: Controlling Charge Transport through Microscopic Devices. Langmuir 2013, 29, 13640-13646.

29. Duan, H.; Wang, D.; Kurth, D. G.; Möhwald, H. Directing SelfAssembly of Nanoparticles at Water/Oil Interfaces. Angew. Chem. 2004, 116, 5757-5760.

30. Samanta, B.; Yang, X.-C.; Ofir, Y.; Park, M.-H.; Patra, D.; Agasti, S. S.; Miranda, O. R.; Mo, Z.-H.; Rotello, V. M. Catalytic Microcapsules Assembled from Enzyme-Nanoparticle Conjugates at Oil-Water Interfaces. Angew. Chem., Int. Ed. 2009, 48, 5341-5344.

31. Glogowski, E.; He, J.; Russell, T. P.; Emrick, T. Mixed Monolayer Coverage on Gold Nanoparticles for Interfacial Stabilization of Immiscible Fluids. Chem. Commun. 2005, 4050-4052. 
32. Glogowski, E.; Tangirala, R.; He, J.; Russell, T. P.; Emrick, T. Microcapsules of PEGylated Gold Nanoparticles Prepared by Fluid-Fluid Interfacial Assembly. Nano Lett. 2007, 7, 389-393.

33. Duan, H.; Wang, D.; Sobal, N. S.; Giersig, M.; Kurth, D. G.; Möhwald, H. Magnetic Colloidosomes Derived from Nanoparticle Interfacial Self-Assembly. Nano Lett. 2005, 5, 949-952.

34. Yogev, D.; Efrima, S. Silver Metal Liquidlike Films (MELLFs). The Effect of Surfactants. Langmuir 1991, 7, 267-271.

35. Collier, C. P.; Saykally, R. J.; Shiang, J. J.; Henrichs, S. E.; Heath, J. R. Reversible Tuning of Silver Quantum Dot Monolayers Through the Metal-Insulator Transition. Science 1997, 277, 1978-1981.

36. Younan, N.; Hojeij, M.; Ribeaucourt, L.; Girault, H. H. Electrochemical Properties of Gold Nanoparticles Assembly at Polarised Liquid|Liquid Interfaces. Electrochem. Commun. 2010, 12, 912-915.

37. Hutter, E.; Fendler, J. H. Exploitation of Localized Surface Plasmon Resonance. Adv. Mater. 2004, 16, 1685-1706.

38. Zhao, L.; Kelly, K. L.; Schatz, G. C. The Extinction Spectra of Silver Nanoparticle Arrays: Influence of Array Structure on Plasmon Resonance Wavelength and Width. J. Phys. Chem. B 2003, 107, 7343-7350.

39. Sandroff, C. J.; Weitz, D. A.; Chung, J. C.; Herschbach, D. R. Charge Transfer from Tetrathiafulvalene to Silver and Gold Surfaces Studied by Surface-Enhanced Raman Scattering. J. Phys. Chem. 1983, 87, 2127-2133.

40. Weitz, D. A.; Lin, M. Y.; Sandroff, C. J. Colloidal Aggregation Revisited: New Insights based on Fractal Structure and Surface-enhanced Raman Scattering. Surf. Sci. 1985, 158, 147-164.

41. Subramanian, V.; Wolf, E. E.; Kamat, P. V. Catalysis with $\mathrm{TiO}_{2} /$ Gold Nanocomposites. Effect of Metal Particle Size on the Fermi Level Equilibration. J. Am. Chem. Soc. 2004, 126, 4943-4950.

42. Siedle, A. R.; Candela, G. A.; Finnegan, T. F.; Van Duyne, R. P.; Cape, T.; Kokoszka, G. F.; Woyciejes, P. M.; Hashmall, J. A. Copper and Gold Metallotetrathiaethylenes. Inorg. Chem. 1981, 20, 2635-2640.

43. Binks, B. P. Particles as Surfactants-Similarities and Differences. Curr. Opin. Colloid Interface Sci. 2002, 7, 21-41.

44. Patra, D.; Sanyal, A.; Rotello, V. M. Colloidal Microcapsules: Self-Assembly of Nanoparticles at the Liquid-Liquid Interface. Chem.-Asian J. 2010, 5, 2442-2453.

45. Milner, S. T.; Joanny, J. F.; Pincus, P. Buckling of Langmuir Monolayers. Europhys. Lett. 1989, 9, 495.

46. Schwartz, H.; Harel, Y.; Efrima, S. Surface Behavior and Buckling of Silver Interfacial Colloid Films. Langmuir 2001, 17, 3884-3892.

47. Bresme, F.; Oettel, M. Nanoparticles at Fluid Interfaces. J. Phys.: Condens. Matter 2007, 19, 413101.

48. Hansen, F. K. Surface Tension by Image Analysis: Fast and Automatic Measurements of Pendant and Sessile Drops and Bubbles. J. Colloid Interface Sci. 1993, 160, 209-217.

49. Du, K.; Glogowski, E.; Emrick, T.; Russell, T. P.; Dinsmore, A. D. Adsorption Energy of Nano- and Microparticles at Liquid-Liquid Interfaces. Langmuir 2010, 26, 12518-12522.

50. Yen, Y.-T.; Lu, T.-Y.; Lee, Y.-C.; Yu, C.-C.; Tsai, Y.-C.; Tseng, Y.-C.; Chen, H.-L. Highly Reflective Liquid Mirrors: Exploring the Effects of Localized Surface Plasmon Resonance and the Arrangement of Nanoparticles on Metal Liquid-Like Films. ACS Appl. Mater. Interfaces 2014, 6, 4292-4300.

51. Lee, K. Y. C. Collapse Mechanisms of Langmuir Monolayers. In Annual Review of Physical Chemistry; Annual Reviews: Palo Alto, 2008; Vol. 59, pp 771-791.

52. Tchoreloff, P.; Gulik, A.; Denizot, B.; Proust, J. E.; Puisieux, F. A Structural Study of Interfacial Phospholipid and Lung Surfactant Layers by Transmission Electron Microscopy after Blodgett Sampling: Influence of Surface Pressure and Temperature. Chem. Phys. Lipids 1991, 59, 151-165.

53. Schultz, D. G.; Lin, X.-M.; Li, D.; Gebhardt, J.; Meron, M.; Viccaro, J.; Lin, B. Structure, Wrinkling, and Reversibility of Langmuir Monolayers of Gold Nanoparticles. J. Phys. Chem. B 2006, 110, 24522-24529.
54. Gopal, A.; Lee, K. Y. C. Morphology and Collapse Transitions in Binary Phospholipid Monolayers. J. Phys. Chem. B 2001, 105, 10348-10354.

55. Takamoto, D. Y.; Lipp, M. M.; von Nahmen, A.; Lee, K. Y. C.; Waring, A. J.; Zasadzinski, J. A. Interaction of Lung Surfactant Proteins with Anionic Phospholipids. Biophys. J. 2001, 81, 153-169.

56. Frens, G. Controlled Nucleation for the Regulation of the Particle Size in Monodisperse Gold Suspensions. Nat. Phys. Sci. 1973, 241, 20-22.

57. Haiss, W.; Thanh, N. T. K.; Aveyard, J.; Fernig, D. G. Determination of Size and Concentration of Gold Nanoparticles from UV-Vis Spectra. Anal. Chem. 2007, 79, 4215-4221.

58. Von Smoluchowski, M. In Handbuch der Electrizität und des Magnetismus (Graetz); Barth: Leipzig, 1921; Vol. 2, p 366.

59. Delgado, A. V.; González-Caballero, F.; Hunter, R. J.; Koopal, L. K.; Lyklema, J. Measurement and Interpretation of Electrokinetic Phenomena (IUPAC Technical Report). Pure Appl. Chem. 2005, 77, 1753-1805. 